\title{
Imaging in Myopia
}

\section{Quan V. Hoang, Jacqueline Chua, Marcus Ang, and Leopold Schmetterer}

Q. V. Hoang

Singapore Eye Research Institute, Singapore, Singapore

Singapore National Eye Centre, Singapore, Singapore

Eye Academic Clinical Program, Duke-NUS Medical School, Singapore, Singapore

Department of Ophthalmology, Harkness Eye Institute, Columbia University Medical Center, New York, NY, USA

J. Chua

Singapore Eye Research Institute, Singapore, Singapore

Singapore National Eye Centre, Singapore, Singapore

Eye Academic Clinical Program, Duke-NUS Medical School, Singapore, Singapore

M. Ang

Singapore Eye Research Institute, Singapore, Singapore

Singapore National Eye Centre, Singapore, Singapore

Eye Academic Clinical Program, Duke-NUS Medical School, Singapore, Singapore

Myopia Centre, Cornea and Refractive Service, Singapore National Eye Centre,

Singapore, Singapore

L. Schmetterer $(\bowtie)$

Singapore Eye Research Institute, Singapore, Singapore

Singapore National Eye Centre, Singapore, Singapore

Eye Academic Clinical Program, Duke-NUS Medical School, Singapore, Singapore

Department of Ophthalmology, Lee Kong Chian School of Medicine, Nanyang Technological University, Singapore, Singapore

School of Chemical and Biomedical Engineering, Nanyang Technological University,

Singapore, Singapore

Department of Clinical Pharmacology, Medical University of Vienna, Vienna, Austria

Center for Medical Physics and Biomedical Engineering, Medical University of Vienna,

Vienna, Austria

e-mail: leopold.schmetterer@seri.com.sg 


\section{Key Points}

- Advances in ocular imaging devices including optical coherence tomography, ultrasound, and magnetic resonance imaging have enabled the visualization of pathologic changes due to myopia, identifying microscopic changes ranging from the posterior segment (sclera, choroid, retina, and/or the optic nerve) to the anterior segment.

- Imaging-derived measures (imaging biomarkers) are potentially useful in assessing degenerative changes occurring in the myopic fundus, in evaluating the early changes preceding myopic macular degeneration, and in providing objective measures of ocular structures to aid in detecting staphyloma formation and progression, myopic macular degenerationtilted disc syndrome, and glaucoma in highly myopic eyes.

- Imaging the highly myopic eye is not straightforward and is associated with challenges such as optics-related aberrations, focusing ability of current devices, and morphological alterations of the myopic fundus and optic nerve.

- An understanding of the imaging of microstructural changes associated with pathological myopia, challenges associated with imaging devices, and potential usefulness and limitations of imaging devices will better inform clinicians of its future potential in the diagnosis and management of vision-threatening complications associated with pathological myopia.

\subsection{Introduction}

In evaluating the myopic eye, imaging has been proven vital in assessing disease complications [1] and prognostication for future management. Early identification of these changes would help predict the development of future complications, which include retinal tears and retinal detachments [2]. Additionally, the possibility of identifying subtle changes in the macula (such as macular schisis or early macular holes; Fig. 10.1) via high-resolution images of the macula could perchance aid clinicians in predicting the development of complications such as macular holes and retinal detachments [3].

Furthermore, imaging may help detect, prognosticate, and guide management of other myopia-related eye diseases. This includes the early detection of atrophy and defects in the basement membrane, located between retinal pigment epithelium (RPE) and Bruch's membrane (BM), which would help predict the risk of developing choroidal neovascularization (CNV) [4] - a leading cause of low vision and blindness in $5-17 \%$ of eyes in patients with pathological myopia (PM) [5]. In addition, imaging is vital for monitoring progression of posterior staphylomas and geographic atrophy, both of which can cause visual impairment in older age groups of patients [6].

Optic disc imaging can also be used to predict the development of glaucoma, where visualization of myopic tilting of the optic disc with peripapillary atrophy and pitting of the optic disc [7], is a possible predisposing factor [8, 9]. Serial imaging investigative measures can therefore be utilized for monitoring the development 

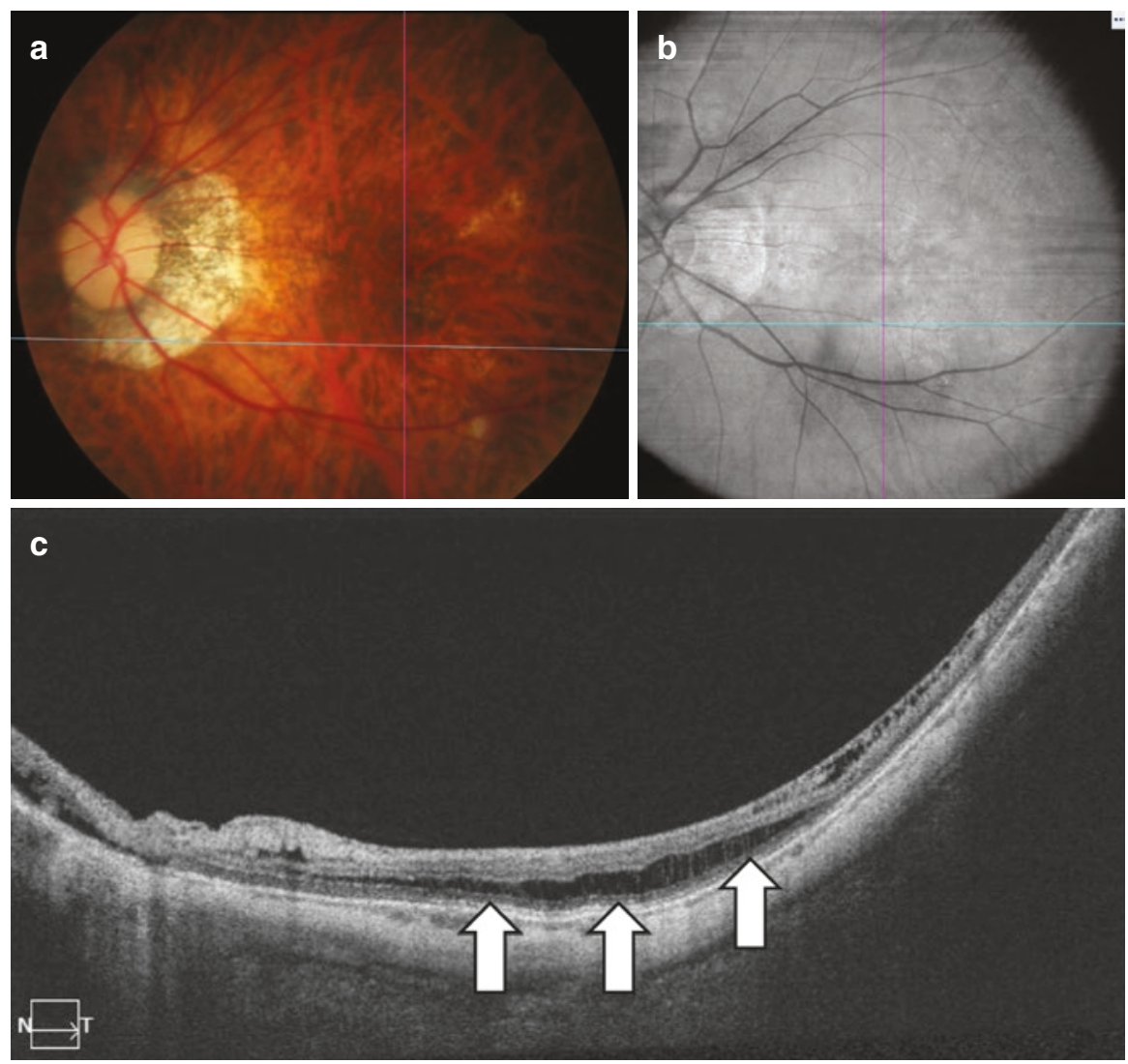

Fig. 10.1 Optical coherence tomography (OCT) imaging findings of a patient with myopic macular degeneration, showing the benefits of using the OCT over color fundus photograph in detecting retinoschisis. (a) Color fundus photograph showing tilted disc with large temporal peripapillary atrophy. (b, $12 \times 12 \mathrm{~mm}$; PLEX Elite, Zeiss) OCT of the fundus showing slight elevation of the retina where the crosshair is positioned. (c) OCT B-scan showing distinct retinoschisis (white arrows)

of open-angle, normal-tension glaucoma [10]. With the hope of exploring novel developments and prospective future clinical applications in the field of imaging in myopia, this chapter thus outlines the significance and challenges of imaging myopic eyes (from the anterior and posterior segments and optic nerve).

\subsection{Disease Characteristics of Myopia}

To best understand how one should approach imaging eyes with myopia, one needs to gear imaging modalities based on the pathophysiology of the disease, which has already been outlined in previous chapters. For example, not only does the sclera of these extremely elongated eyes show marked scleral thinning [12, 13], some eyes thin to the extent that local outpouchings or staphylomas form (Figs. 10.2 and 10.3). Staphylomas are often recognized as the harbinger of PM, reported in up to $90 \%$ of 

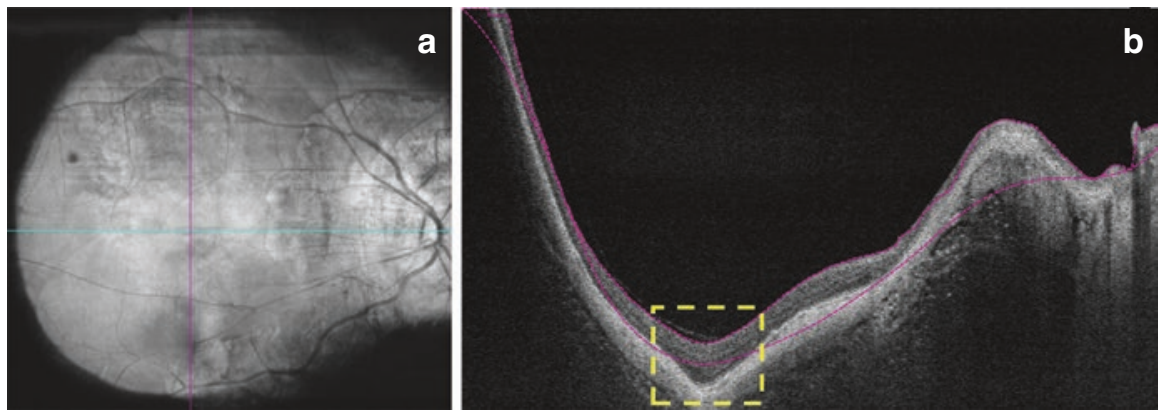

Fig. 10.2 Optical coherence tomography (OCT) imaging findings of a highly myopic patient, showing the benefits of using the OCT over fundus photograph to detect posterior staphyloma. (a, $12 \times 12 \mathrm{~mm}$; PLEX Elite, Zeiss) OCT fundus photograph showing tilted disc with large temporal peripapillary atrophy. (b) OCT B-scan showing distinct posterior staphyloma (yellow dotted box)

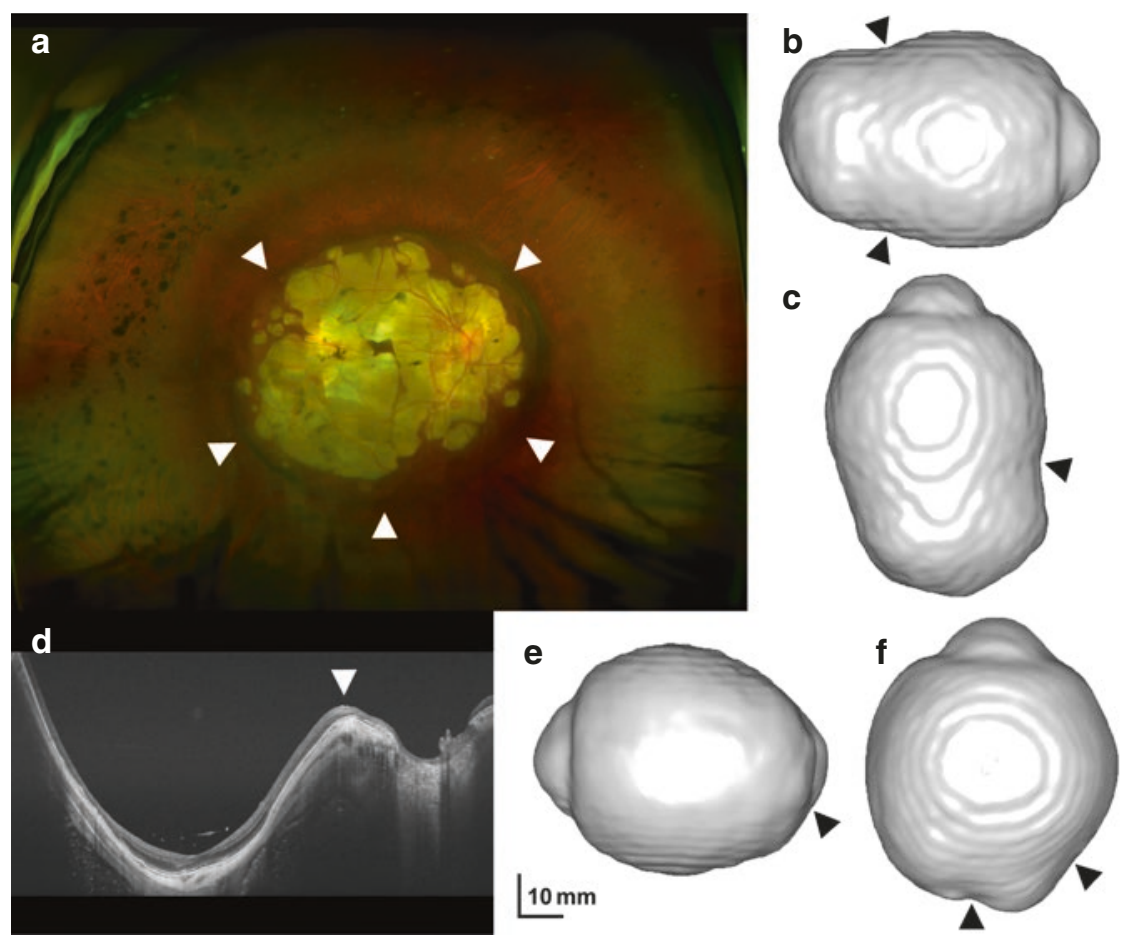

Fig. 10.3 Fundus photography, swept source optical coherence tomography (SSOCT), and 3-dimensional magnetic resonance imaging (MRI) of patients with posterior staphyloma. Patient 1 $(\mathbf{a}, \mathbf{b}, \mathbf{c})$ was a 58-year-old woman with an axial length of $35.4 \mathrm{~mm}$ in the right eye (OD). The right fundus shows a deep, circular-shaped posterior staphyloma, with an abrupt change in scleral curvature (arrowheads) apparent on fundus photography (A) and OD MRI volume renderings (B- temporal view, C- inferior view). Patient 2 (d, e, f) was a 61-year-old woman with an OD axial length of 33.8 $\mathrm{mm}$. A posterior staphyloma was present, with an abrupt change in scleral curvature (arrowheads) apparent on OD SSOCT (D) and OD MRI volume renderings (E- nasal view, F- superior view) 

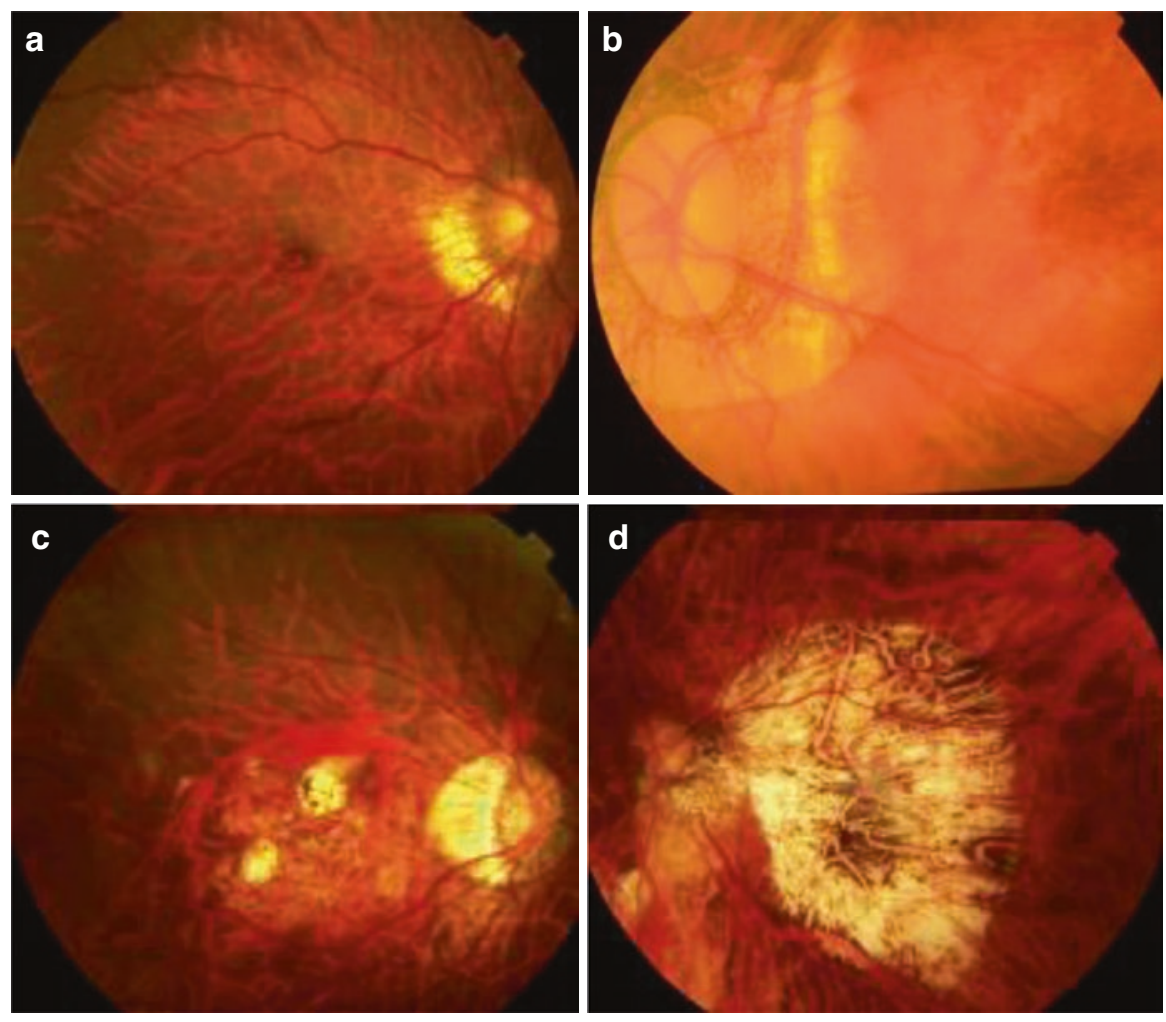

Fig. 10.4 Color fundus photographs showing the worsening levels of myopic macular degeneration; (a) Category 1, (b) Category 2, (c) Category 3 (c), and (d) Category 4

high myopia (HM) patients $[14,15]$. More recent reports noted a severe grade of staphyloma being associated with further eye elongation [16] and more severe and progressive myopic macular degeneration (MMD; Fig. 10.4) [14, 17, 18]. Given that MMD is the leading cause of blindness in Japan [19], and the second leading cause in Chinese adults [20], it highlights the importance of closely monitoring patients with a severe grade of staphyloma. Risk of vision loss from myopic maculopathy (e.g., from splitting of retinal layers (foveoschisis), hole formation, atrophy, and CNV) may correlate with the specific location and conformation [11] of staphylomas [21].

\subsection{Key Structures Altered in Myopia and Pathological Myopia}

\subsubsection{Sclera and Collagen}

Changes in sclera and collagen structures may underlie axial elongation and staphyloma formation and progression. The anatomical changes underlying global eye elongation and local staphyloma formation likely occur in the component collagen 
fibers of the sclera. In mammalian models, there is scleral thinning and tissue loss during myopia development $[11,22]$ with a net decrease in scleral collagen (with decreased collagen synthesis and increased degradation) evidenced by reduced dry weight and hydroxyproline content $[23,24]$. Decreased collagen fiber diameter and decreased collagen crosslinking (CXL) are seen in both mammalian models and HM patients $[13,25]$. Specifically, in the guinea pig myopia model, there is scleral remodeling, possibly from greater slippage [26] between collagen fibrils and/or collagen fiber bundles due to fibroblast deactivation, with decreased expression of type I collagen, and $\alpha_{2}$ and $\alpha_{1}$ integrin $[27,28]$. In the tree shrew mammalian myopia model, blockage of collagen crosslinking with $\beta$-aminopropionitrile resulted in an increased degree of myopia-induced vitreous elongation and scleral thinning at the posterior pole [29]. Moreover, the development of high myopia and pathologic myopia may be driven by genes such as the scleral remodeling gene LAMA2, leading to scleral thinning and staphyloma formation [30].

\subsubsection{Choroidal Changes}

Choroidal changes in myopia may contribute to atrophy and myopic macular degeneration. Morphological change such as the thinning of the choroidal vascular layer is closely associated with increasing levels of myopia and MMD (either immediately preceding, concurrent with or immediately following the development of MMD) [31-33]. Specifically, Wei et al. reported that subfoveal choroidal thickness in their Beijing-based cohort decreased by $15 \mu \mathrm{m}$ per diopter of increased myopia [31]. There are various lesions comprising myopic maculopathy [3, 11, 18, 34, 35] that all involve the choroid, namely, diffuse chorioretinal atrophy, patchy chorioretinal atrophy, macular atrophy (bare sclera), lacquer cracks, and myopic choroidal neovascularization. Diffuse atrophy is a yellowish, ill-defined lesion [34], and is characterized by a marked thinning of the choroid as seen on optical coherence tomography (OCT), but with relatively preserved outer retina and retinal pigment epithelium, allowing for relatively good vision. Patchy chorioretinal atrophy is whitish, well-defined atrophy [34] with complete localized loss of the choroid with only the large choroidal vessels sporadically remaining within the atrophic area. Loss of the choroid is soon followed by loss of outer retina and retinal pigment epithelium. Macular atrophy is similar to patchy atrophy but tends to be centered at the fovea.

\subsubsection{Bruch's Membrane and Retinal Pigment Epithelium Changes}

The hallmark anatomical changes that occur alongside the development and progression of MMD are changes in BM and RPE as reflected in increasing degrees of atrophy [36]. Moreover, BM changes have been postulated to be the main driver of axial elongation overall [37]. Recently, Ohno-Matsui et al. reported the use of swept-source OCT (SS-OCT) to demonstrate that patchy atrophy was not 
simply chorioretinal atrophy, but also included a hole in Bruch's membrane [38]. Angiographically observed vessels within the area of patchy atrophy may include large choroidal vessels, intrascleral vessels, and retrobulbar vessels. Lacquer cracks are observed as yellowish linear lesions [34, 39, 40], and represent the mechanical rupture of BM [41]. Jonas et al. have reported an increase in disc-fovea distance due to an increase in the peripapillary gamma zone. Since BM thickness was found to be independent of axial length [42], even in the much larger surface area in an axially elongated eye in a human histomorphometric study, Jonas et al. inferred that the volume of BM increased with longer axial length and BM is actively produced during axial length elongation [37]. They postulated that BM was a primary driver of axial elongation that led to choroidal thinning through "compression." If this was indeed the mechanism, then the RPE is an ideal target for medical intervention in both eyes that are already severely elongated, as well as those undergoing progression, but still only minimally elongated.

\subsection{Existing Imaging Modalities to Evaluate the Myopic Eye}

\subsubsection{Optical Coherence Tomography}

Ophthalmic OCT systems can image the anterior segment as well as the posterior segment of the eye such as the retina, BM, RPE, and choroid. Spectral-domain OCT (SD-OCT, Spectralis, Heidelberg Eng., Carlsbad, CA) provides an axial resolution of $4 \mu \mathrm{m}$ and a lateral resolution of $14 \mu \mathrm{m}$, but only about $1 \mathrm{~mm}$ of penetration into the retina. The extremely fine resolution of OCT allows better assessment of retinal layers such as photoreceptors, the ganglion cells, plexiform, and nuclear layers [43] and vitreous membranes in proximity to the retina. In contrast to the SD-OCT, SS-OCT uses a tunable laser with a longer wavelength of $1050 \mathrm{~nm}$, which achieves greater penetration of tissue with less signal roll-off, allowing better visualization of choroidal anatomy [38, 44, 45] and has specifically been employed to correlate defects in BM with the atrophy of MMD [38]. In addition to utilizing choroidal thickness as biomarker for myopia progression [31-33], recent developments provide further insight into the function of the choroid, including the calculation of choroidal vascular index (CVI, the vessel-area-to-stromal-area ratio using a validated automated algorithm) [46] and OCTA using the SS-OCT (SS-OCTA, a noninvasive method of imaging choroidal vasculature, and specifically the foveal avascular zone (FAZ) without the administration of intravenous dye) [47, 48]. Newer UWF-OCT systems allow for even wider $\left(100^{\circ}\right)$ scans. Wider scans are likely crucial to accurately depicting staphyloma shape [49].

\subsubsection{Ultrasound}

Ophthalmic ultrasound can image the entire eye dynamically. Since their introduction in 1958, mechanically scanned, single element $10 \mathrm{MHz}$ transducers have largely been 
the norm for imaging of the eye [50]. UBM systems (Escalon-Sonomed, Quantel, Ophthalmic Technologies, among others) have been used for diagnostic imaging and biometric characterization [51, 52]. Systems operating at $20 \mathrm{MHz}$ were introduced (Quantel Medical, Bozeman, MT) for examination of the posterior segment of the eye and the vitreoretinal interface $[51,53]$. With an axial resolution of about $75 \mu \mathrm{m}$, these systems provide superior resolution compared to conventional $10 \mathrm{MHz}$ systems as well as the ability to image the retina. They also provide far deeper penetration than optical modalities (10 mm vs. $1 \mathrm{~mm}$ for OCT). Commercial single-element systems are designed such that during a contact examination, the geometric focus of the transducer falls just anterior to the retina. However, the anterior vitreous and anteriorsegment structures (cornea, iris, ciliary body, and lens) are poorly visualized because they fall within the unfocused near field of the transducer which makes current ophthalmic systems unsuitable for imaging the entire vitreous. Recent advancements in annular-array imaging technology overcome these technical limits [54].

\subsubsection{Magnetic Resonance Imaging}

MRI allows for imaging of the whole eye (Fig. 10.3). The standard ophthalmology imaging modalities of ultrasound and optical coherence tomography are limited by a relatively narrow field of view. MRI does allow for imaging of the entire eye globe, but visualizing eye wall distortions at standard MR resolutions minimizes effect sizes and induces aliasing artifacts that obfuscate distortions. Super-resolution is an established MR approach that provides a means of detecting extremely small distortions by increasing signal to noise (with isotropic resolutions down to 50-100 $\mu \mathrm{m}$ ), reducing scan time (which would allow patients to more readily maintain fixation, thereby reducing eye movement artifacts), and minimizing aliasing artifacts that can bias estimates $[55,56]$.

\subsection{Challenges in Imaging of the Myopic Eye}

\subsubsection{Interaction Between Low- and High-Order Aberrations}

Given the presence of two positive lenses (the cornea and the crystalline lens) in the eye, any imaging of intraocular structures is contingent on their properties. This makes direct visualization and imaging structures in the posterior segment, secondary to optical imperfections through the cornea and lens, the main challenge in imaging a myopic eye. Examples of such would include light diffraction in the pupil, intraocular scattering, and optical aberrations. As a result of these imperfections, imaging of patients with high myopia using any modality (such as fundus photography) would have a poorer image quality due to an imbalance of low- and high-order aberrations $[57,58]$. Other causes to consider include the limitations of focusing lenses used by the imaging device. For example, the device may not be able to compensate (diopter compensation) sufficiently in patients with very high myopia (more than $-12.0 \mathrm{D}$ ). Pathological myopia is also associated with varying 
structural changes within the eye (due to the abnormal eye elongation, scleral and corneal curvature irregularities, cataracts leading to poor clarity, or retinal thinning causing abnormal projections of the final image $[58,59])$. Such structural changes are demonstrated in altered optic nerve head morphology.

Taking into considerations the challenges faced when imaging the myopic eye, current standards of practice for investigations include fundus photography, dyebased angiography, ultrasound (including biomicroscopy), and magnetic resonance imaging (MRI). The OCT has also become an often-used tool for the characterization of ocular tissue structural changes arising from myopia [60]. Swept source (SS)-OCT has a higher wavelength of $1050 \mathrm{~nm}$ and is hence able to penetrate into deeper layers. It is also capable of wide-angle scans, given its speed of capture and software engineering. More recently, OCT angiography (OCTA) has enabled visualization of the vasculature of the eye [61-63]. Nonetheless, there still exists room for improvement and various constraints. In the case of wide-field OCT (WF-OCT), problems with segmentation in the periphery may arise due to inadequate depth range to image the complete anterior-posterior extent of the posterior pole. Further considerations arise regarding the normative databases of OCT systems, where reference and patient cohorts should ideally be derived from the same population to reflect similar comorbidities [64]. Stratifications normally employed for covariates that affect measurements (such as age, ethnicity, and refractive error) are challenging to derive in myopic eyes - a result of large regional variation in refractive error prevalence [65]. In regions with a high prevalence of myopia and pathological myopia, this results in normative databases being poor representations of the existing patient population.

\subsubsection{Challenges in Imaging the Anterior Segment}

In high myopia, while most refractive errors in myopic eyes arise from an axial length, alterations in the anterior segment lead to significant optical and high-order aberrations [66]. Furthermore, the association between myopia and biomechanical changes in the cornea has implications in the assessment of intraocular pressure and may have a role in the pathogenesis of myopia [67]. Indeed, existing studies report inconsistent results - attributable to different imaging and measurement techniques, and the technological limitations in measuring biomechanical changes in the cornea associated with increasing myopia [68]. Preoperative clinical evaluation of patients with high myopia still necessitates imaging of the cornea and anterior segment, given their increasing risk of developing ectasia proportionate to the amount of corneal tissue removed in laser refractive surgeries [69]. Furthermore, as high myopes are more likely to require phakic lens implantation [70], accurate anterior segment imaging is necessary to ensure adequate safety measures prior to implantation and accurate intraocular lens (IOL) implantation. Especially in high myopes, accurate formulae, anterior segment imaging, and corneal topography are all necessary to prevent refractive surprises [71] and complications postoperatively. Significant improvements in IOL calculations and refractive prediction in high myopes [71] 
have fortunately been accomplished with new developments in biometry incorporating anterior segment OCT technology [72], coupled with next-generation formulae.

\subsubsection{Challenges in Imaging the Retina}

Prior to the discovery of modern imaging techniques, there were three main challenges faced in retinal imaging. First, the diagnosis of myopic foveoschisis was challenging based on clinical examination and fundus photography due to the lack of contrast between the retinal tissues and underlying choroid. Second, posterior staphylomas, a hallmark of pathological myopia [73], could not be identified from standalone two-dimensional fundus photography with ease. Finally, it is also challenging to capture lattice degeneration and retinal breaks in the peripheries on traditional $50^{\circ}$ fundus photographs.

With the OCT, the way clinicians diagnosed and managed retinal complications of pathological myopia was transformed-with the ability to examine retinal layers in vivo and at high resolution. The ability to view distinct retinal layers has enhanced visualization of myopic traction maculopathy (MTM), for which splitting of the retinal layers can be well visualized on cross-sectional OCT scans of the retina. This can be taken a step further using retromode fundus imaging [74]. Examples of features that can be seen include inner or outer retinal schisis, foveal detachment, lamellar, or full thickness macular hole and/or macular detachment $[75,76]$.

Another aspect in which OCT has advanced the diagnosis and management of myopia-related eye diseases is in the characterization of posterior staphylomas, which are one of the hallmarks of pathological myopia. Nonstereoscopic fundus photographs are inadequate for detailed studies of posterior staphylomas as the change in contour at the staphyloma edge is not always discernible. The OCT overcomes this limitation because of its excellent depth resolution [21, 77]. Comparing their capability in visualizing posterior staphylomas, Shinohara et al. showed no significant differences between using WF-OCT and three-dimensional (3D) MRI imaging. It is noted, however, that ultrawide-field OCT (UWF-OCT) had the advantage in visualizing the spatial relationship of the staphylomas with the optic nerve and macula $[78,79]$.

Comparing the use of conventional fundus photography to UWF retinal imaging, the former has been shown capable of imaging up to $50^{\circ}$ of the retina, while the latter covers a wider field of the retina at $200^{\circ}$. Given the high prevalence of lattice degeneration and retinal breaks in the peripheral retina in patients with highly myopic eyes, the advantages of UWF retinal imaging can be appreciated.

A potentially blinding complication of high myopia, myopic choroidal neovascularization $(\mathrm{mCNV})$ is not easily diagnosed and monitored solely with clinical examinations. Multimodal imaging thus has a pertinent place in assessing mCNV. Verifying the diagnosis of mCNV requires the use of a fundus fluorescein angiography (FFA) where leakage is seen as increasing size and intensity of hyperfluorescence with time [80]. A feature of pathological myopia, retinal avascularity in the $360^{\circ}$ of the periphery, can also be revealed using wide-field FFA [81]. Distinguishing the myriad 
causes of subretinal exudation in high myopes (i.e., simple lacquer crack hemorrhage, inflammatory lesions, and $\mathrm{mCNV}[82,83]$ ) can be achieved with OCTA, which has the ability to identify neovascular membranes noninvasively. However, FFA remains the gold standard for mCNV diagnosis, given OCTA's comparatively lower sensitivity [84]. Furthermore, FFA is still necessary for monitoring disease activity, as OCTA lacks the ability to discern disease activity — where the flow signal may persist in an inactive mCNA [83-85]. Still, OCTA can assist with distinguishing the stages of mCNV via identification of signs of activity. These include illdefined margins [86], disruption of the external limiting membrane [87], and variable amounts of intraretinal and subretinal fluids. These hyper-reflective lesions coalesce and develop a distinct border with appropriate treatment. Previous studies using the OCTA have suggested that rupture of the Bruch's membranes may be the cause of macular atrophy developing after $\mathrm{mCNV}$ [88]. However, the combination of OCTA and SS-OCT in multimodal imaging has given promising results-suggesting that $\mathrm{mCNV}$ is directly supplied by the short posterior ciliary artery instead of the choroidal vasculature as previously postulated [89].

\subsubsection{Challenges in Imaging the Choroid and Sclera}

Lying between the sclera and the RPE, the choroid's anatomical location makes imaging a challenge without the use of methods such as indocyanine green (ICG) angiography, ultrasonography (US), and OCT. Moreover, the choroid in pathological myopia is often extremely thin and thus difficult to measure. The addition of other, varied factors that interfere with choroid imaging in the myopic eye further complicate the process.

Typical fluorescein angiography has limited use in choroidal visualization since melanin impedes most of the spectra emitted from the agent. Imaging is also made near impossible with light absorption and scattering produced by the RPE pigment and choroidal blood. On the other hand, angiography via ICG has better use for the evaluation of various choroidal vasculopathy and inflammatory diseases [90]. This can be attributed to ICG's emitted spectrum being within the near-infrared wavelength — which is not disrupted by the RPE pigment or choroidal blood [91]. Nonetheless, other modalities such as OCT and autofluorescence imaging are still more feasible in the clinical setting and are thus still more often used than ICG angiography. Other modes of imaging include OCTA for assessing choroidal vasculature and its structures [46-48], as well as B-scan ultrasound to image the choroid with better penetration. However, B-scan images provide poorer resolution than those of OCTA - which is subsequently worsened by the thinning of the choroid in PM [53]. Yet, despite its flaws, the ultrasound remains useful in detecting staphylomas and globe contours.

With regard to OCT, we find that spectral domain OCT (SD-OCT) instruments with enhanced depth imaging can visualize the choroid more readily [92]. Inadequacies still exist in OCT images of the choroid, where deeper tissues imaged result in poorer sensitivity. The signals obtained in the choroid are also usually weak due to the high 
attenuation coefficient of the RPE. Nonetheless, ironically due to thinner choroids and relative depigmentation, full thickness imaging of the choroid is still attainable in high myopes. Yet, OCT image quality may still be compromised by distortions caused by the likely presence of staphylomas and abnormal eyewall contours in these same patients with high myopia. On the other hand, the extent of decreased sensitivities with increased tissue depth is comparatively lower in SS-OCT [93]. Clearly, notwithstanding the technique used, many challenges and limitations still exist in OCT imaging of high myopia - arising from the extreme axial length of highly myopic eyes, curvature deformity exaggeration on OCT, and the production of imaging artifacts [49].

The inability of OCT to readily distinguish the posterior scleral boundary makes scleral imaging a greater challenge than choroid imaging-where the choroid itself (unless extremely thin) reduces scleral image quality by disrupting signals. With the use of "Reflectivity" software, scleral boundaries may be augmented via adaptive compensation based on direct application of pixel intensity exponentiation. This would improve the precision of scleral thickness assessment [94, 95]. In retrospect, we conclude that the use of 3D MRI and ultrasound have more favorable results in visualizing scleral contours and characterizing staphylomas. Using animal experimentations on guinea pigs ex vivo and humans in vivo, we were able to demonstrate that quantitative ultrasound and super-resolution 3D MRI can be used to localize areas of scleral weakness and elasticity. These modalities could potentially detect scleral weakness preceding staphyloma formation; thereby discerning and even predicting which highly myopic eye is destined to develop staphylomas and MMD. This is critical in research studies targeted toward scleral reinforcementsuch as macular buckling [96] or scleral collagen crosslinking [97, 98].

\subsubsection{Challenges in Imaging the Optic Nerve}

Features such as optic disc tilt (Fig. 10.5), peripapillary atrophy (PPA), and abnormally large or small optic discs are the earliest known structural alterations that potentially predict the development of pathological myopia, and can be observed even in young highly myopic adults. Unfortunately, these features (some also with associations to glaucoma) also interfere with the visualization of optic disc margins $[99,100]$, and are also not easy to discern in highly myopic eyes [101]. There is also added difficulty in eyes with myopic maculopathy, where visual field defects result in further interference [102]. As such, the answer to these challenges may lie in imaging deep optic nerve head structures (such as parapapillary sclera, scleral wall, and lamina cribrosa) [103] in highly myopic eyes for more precise diagnoses of glaucoma.

Glaucomatous optic nerve damage may be worsened by obstructed axoplasmic flow within the optic nerve head-possibly caused by a disrupted lamina cribrosa (LC) arising from optic disc tilt [104]. Imaging performed to evaluate the integrity of the lamina cribrosa may, therefore, aid in prognosticating risk for eventual glaucoma development. Indeed, Sawada et al. have shown via enhanced depth OCT imaging that LC defects in myopic eyes (with and without primary open-angle glaucoma) are associated with optic disc tilt angle, which can also explain the location and severity of glaucomatous visual field defects [104]. 

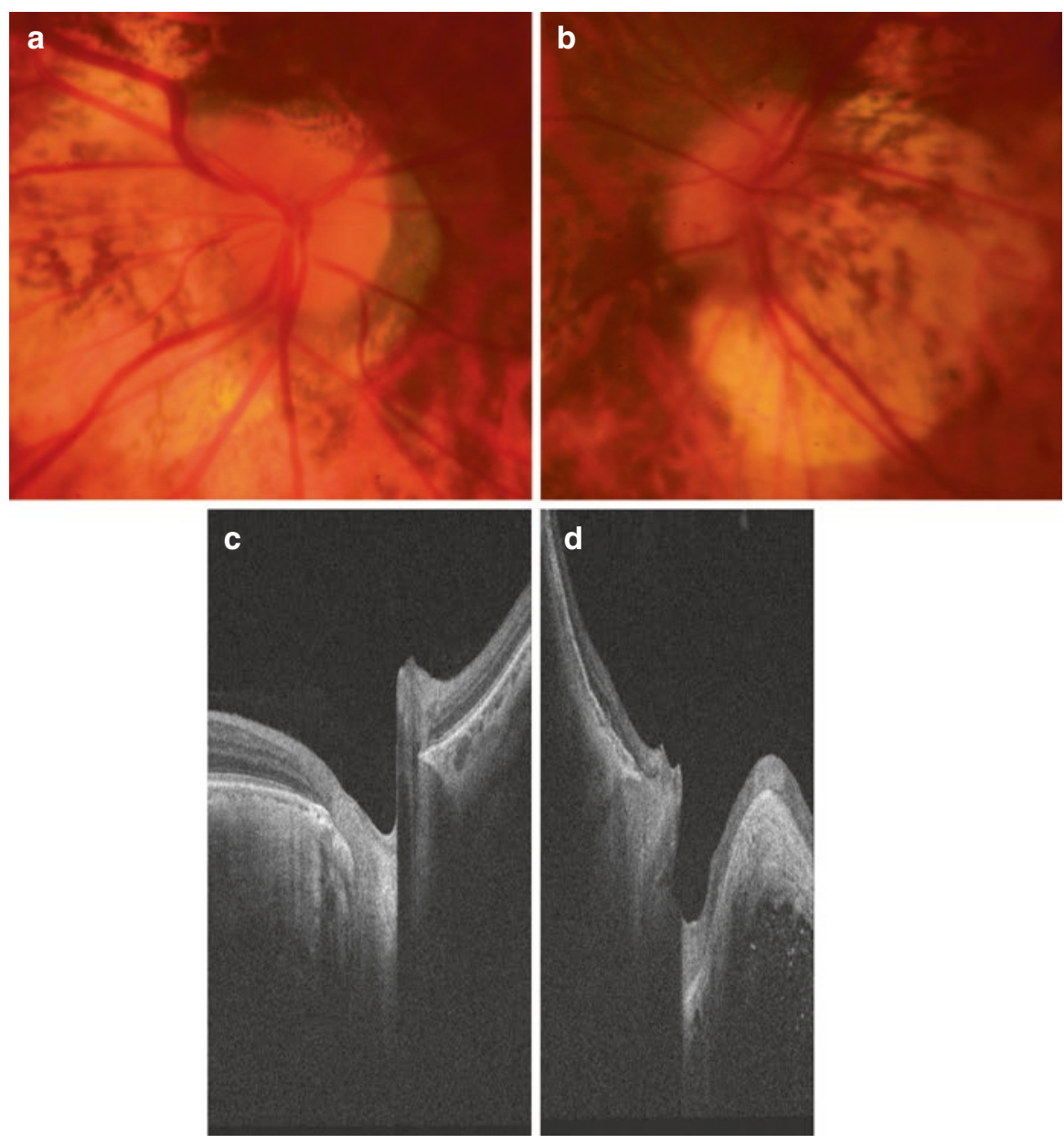

Fig. 10.5 Color photographs (top panels) and optical coherence tomography (bottom panels) of myopic tilted optic nerve discs showing moderately-tilted discs (left panels) and a severely tilted discs (right panels)

The optic nerve exits the eye at the Bruch's membrane opening (BMO), which can be easily discerned on OCT imaging. Using conventional disc margin-based measurements as a standard for comparison, Malik et al. investigated the utility of the BMO as a possible landmark for neuroretinal rim measurements, and has shown the BMO minimum rim width (BMO-MRW) to be markedly more sensitive (71\% against $30 \%$ ) than disc margin rim area (DM-RA) in diagnosing glaucoma [105].

Meanwhile, Enders et al. further demonstrated that a two-dimensional neuroretinal rim parameter based on BMO_namely, the BMO minimum rim area (BMOMRA), outclassed all other measures (such as BMO-RMW, retinal nerve fiber layer thickness, and DM-RA) in diagnosing glaucoma [106]. Unfortunately, the diagnostic performance of this parameter has yet to be validated in myopic eyes. 
Another deep optic nerve head structure that can be imaged on the enhanced depth OCT is the border tissue of Elschnig — a cuff of collagenous tissue arising from the sclera and joining the Bruch's membrane at the optic disc margin [104]. Having reviewed the externally oblique border tissue length (EOBT), optic nerve head tilt angle and optic canal obliqueness, Han et al. have concluded that temporally located maximal values for these parameters were both independently associated with the presence of myopic normal tension glaucoma and consistent with the location of retinal nerve fiber layer defects [103].

Macular ganglion cell complex (GCC) thickness has been reported to have comparable diagnostic power to circumpapillary retinal nerve fiber layer (cp-RNFL) thickness [107-110] — which on its own is strongly suggestive of glaucoma in nonmyopic eyes, but faces limitations in interpretation in high myopes with PPA. Zhang et al. have indeed demonstrated that macular GCC parameters are superior to cpRNFL parameters in diagnosing high myopes with glaucoma-with focal loss volume (FLV) on RTVue-OCT and minimum ganglion cell-inner plexiform layer (GCIPL) on the Cirrus HD OCT having the best diagnostic power among these macular parameters [111]. Yet, two obstacles persist in limiting the use of macular GCC measurements for the diagnosis of glaucoma in high myopes. Namely, these are the lack of a normative database in highly myopic eyes, as well as inaccurate measurements attributable to choroidal atrophy in myopic maculopathy. New developments regarding the use of OCTA on the optic nerve have suggested that it is a prospective adjunctive tool for evaluating the myopic disc for glaucomatous changes. Suwan et al. measured the perfused capillary density (PCD) via a $4.5 \times 4.5 \mathrm{~mm}$ OCTA scan centered on the optic nerve head and has showed that eyes with myopia and open angle glaucoma had the lowest PCD compared to eyes with glaucoma alone and control eyes [112].

\subsection{Future Developments}

The primary aim of imaging in myopia would be to study the tissues involved with the pathological elongation of the eye, namely, the sclera as well as the choroid that is the regulator of scleral extracellular matrix remodeling [113], and its biomechanical properties [114]. As the RPE is highly scattering, it makes imaging of the myopic eye highly challenging. To make matters worse, the choroid is highly vascularized and perfused with a high concentration of red blood cells, this also causes a significant amount of light scattering making imaging more complicated [115]. One way to circumvent this problem would be to use OCT systems with wavelengths around $1060 \mathrm{~nm}$ to image the choroid [116]; however, the results obtained from this mode are less than satisfactory due to its relatively poor resolution capacity and inability to visualize the microvasculature and cellular structure of the choroid. Due to the complexity of this issue, there is no sensible nomenclature available to classify choroidal layers and boundaries, nor a definition of the choroidal-scleral interface [116]. As such, to facilitate comparisons and meta-analysis of existing 
data, there is a desperate need for a standardization for choroidal segmentation and OCT-based definition of the choroidal layers.

The OCT itself has its shortcomings: the sclera cannot be visualized using the OCT, higher wavelengths (around $1300 \mathrm{~nm}$ range) used for anterior segment OCT would not be able to be used to image the posterior pole either due to the high absorption coefficient of water contained in the vitreous [72]. These limitations also extend to the use of OCTA. There is currently no standard protocol for segmentation, the outcome parameters for OCTA have not been clearly defined either. Although some authors have tried to use analysis of flow voids or signal voids in the choriocapillaris to quantify the area taken up by the microvasculature [117, 118], the data pertaining to myopic patients are but insufficient [119]. Moreover, different authors have used different image analysis methods to quantify signal voids, as such, there is no clear common protocol in place to address the underlying issue. Looking into the future, there is however incipient research suggesting that the comprehension of blood supply and changes in vasculature from the anterior to the posterior segment of the myopic eye is crucial to the understanding of the disease [120-123].

Photoacoustic imaging has shown promise recently to fill the gaps between OCT and ultrasound in terms of penetration depth. Ultrasound biomicroscopy (higher ultrasound frequencies used) of the anterior segment simply does not have enough penetrative depth to image the posterior pole of the eye [124]. Photoacoustic imaging detects the waves generated by the absorption of pulsed laser light in tissues [125]; meanwhile, other modalities use contrast solely based on absorption. This modality has been used before to image the posterior pole of the eye in vitro and in animal models in vivo. This can also be used in concurrence with angiography, measuring oxygen saturation and pigment imaging [126]. However, there are some limitation pertaining to this modality notwithstanding moderate depth resolution, pure optical absorption sensing, need for contact detection with ultrasound sensor, and a relatively long acquisition time. In view of these limitations, we have yet to receive tangible results from photoacoustic imaging for posterior pole imaging in humans.

Recently, there has been a shift to elastography approaches to study the biochemical properties of ocular tissues with either OCT [127] or with ultrasound [128], the visualization of biochemical properties of the eye coats in vivo would otherwise be incredibly challenging This perspective is propelled by several studies proposing the measurement of ocular rigidity using noninvasive measures instead $[129,130]$. However, none of these studies have addressed or included patients with myopia. The crux of this technique pertains to using an internal or external force to induce movement of tissue that is subsequently picked up using imaging devices, most in vivo work would be aimed at biochemical properties of the cornea $[131,132]$. In vivo imaging using an ocular pulse with an internal excitation source is not yet able to produce results of biochemical parameters [133, 134]. Meanwhile, some in vivo work has been pursued involving the use of acoustic radiation force optical coherence elastography [135]. 


\section{References}

1. Chen $\mathrm{L}$, et al. Rhegmatogenous retinal detachment due to paravascular linear retinal breaks over patchy chorioretinal atrophy in pathologic myopia. Arch Ophthalmol. 2010;128(12):1551-4.

2. Mitry D, et al. The epidemiology of rhegmatogenous retinal detachment: geographical variation and clinical associations. Br J Ophthalmol. 2010;94(6):678-84.

3. Hayashi K, et al. Long-term pattern of progression of myopic maculopathy: a natural history study. Ophthalmology. 2010;117(8):1595-611.

4. Yoshida T, et al. Myopic choroidal neovascularization: a 10-year follow-up. Ophthalmology. 2003;110(7):1297-305.

5. Ikuno Y, et al. Ocular risk factors for choroidal neovascularization in pathologic myopia. Invest Ophthalmol Vis Sci. 2010;51(7):3721-5.

6. Ohno-Matsui $\mathrm{K}$, et al. Intrachoroidal cavitation in macular area of eyes with pathologic myopia. Am J Ophthalmol. 2012;154(2):382-93.

7. Samarawickrama $\mathrm{C}$, et al. Myopia-related optic disc and retinal changes in adolescent children from singapore. Ophthalmology. 2011;118(10):2050-7.

8. Jonas JB, et al. Histology of the parapapillary region in high myopia. Am J Ophthalmol. 2011;152(6):1021-9.

9. Park HY, Lee K, Park CK. Optic disc torsion direction predicts the location of glaucomatous damage in normal-tension glaucoma patients with myopia. Ophthalmology. 2012;119(9):1844-51.

10. Marcus MW, et al. Myopia as a risk factor for open-angle glaucoma: a systematic review and meta-analysis. Ophthalmology. 2011;118(10):1989-1994.e2.

11. Curtin BJ. The myopias: basic science and clinical management. Philadelphia: Harper and Row; 1985.

12. Curtin BJ, Teng CC. Scleral changes in pathological myopia. Trans Am Acad Ophthalmol Otolaryngol. 1958;62(6):777-88.

13. Avetisov ES, et al. A study of biochemical and biomechanical qualities of normal and myopic eye sclera in humans of different age groups. Metab Pediatr Syst Ophthalmol. 1983;7(4):183-8.

14. Hsiang HW, et al. Clinical characteristics of posterior staphyloma in eyes with pathologic myopia. Am J Ophthalmol. 2008;146(1):102-10.

15. Curtin BJ, Karlin DB. Axial length measurements and fundus changes of the myopic eye. I. The posterior fundus. Trans Am Ophthalmol Soc. 1970;68:312-34.

16. Saka N, et al. Changes of axial length measured by IOL master during 2 years in eyes of adults with pathologic myopia. Graefes Arch Clin Exp Ophthalmol. 2013;251(2):495-9.

17. Lin LL, et al. Epidemiologic study of the prevalence and severity of myopia among schoolchildren in Taiwan in 2000. J Formos Med Assoc. 2001;100(10):684-91.

18. Steidl SM, Pruett RC. Macular complications associated with posterior staphyloma. Am J Ophthalmol. 1997;123(2):181-7.

19. Iwase A, et al. Prevalence and causes of low vision and blindness in a Japanese adult population: the Tajimi Study. Ophthalmology. 2006;113(8):1354-62.

20. Saka N, et al. Long-term changes in axial length in adult eyes with pathologic myopia. Am J Ophthalmol. 2010;150(4):562-568.e1.

21. Ohno-Matsui K, et al. Association between shape of sclera and myopic retinochoroidal lesions in patients with pathologic myopia. Invest Ophthalmol Vis Sci. 2012;53(10):6046-61.

22. McBrien NA, Cornell LM, Gentle A. Structural and ultrastructural changes to the sclera in a mammalian model of high myopia. Invest Ophthalmol Vis Sci. 2001;42(10):2179-87.

23. McBrien NA, Lawlor P, Gentle A. Scleral remodeling during the development of and recovery from axial myopia in the tree shrew. Invest Ophthalmol Vis Sci. 2000;41(12):3713-9.

24. Norton TT, Rada JA. Reduced extracellular matrix in mammalian sclera with induced myopia. Vis Res. 1995;35(9):1271-81.

25. Wollensak G, Iomdina E. Crosslinking of scleral collagen in the rabbit using glyceraldehyde. J Cataract Refract Surg. 2008;34(4):651-6. 
26. McBrien NA, Jobling AI, Gentle A. Biomechanics of the sclera in myopia: extracellular and cellular factors. Optom Vis Sci. 2009;86(1):E23-30.

27. Wang Q, et al. Form-deprivation myopia induces decreased expression of bone morphogenetic protein-2, 5 in guinea pig sclera. Int J Ophthalmol. 2015;8(1):39-45.

28. Tian XD, et al. Expressions of type I collagen, alpha2 integrin and beta1 integrin in sclera of guinea pig with defocus myopia and inhibitory effects of bFGF on the formation of myopia. Int J Ophthalmol. 2013;6(1):54-8.

29. McBrien NA, Norton TT. Prevention of collagen crosslinking increases form-deprivation myopia in tree shrew. Exp Eye Res. 1994;59(4):475-86.

30. Li YT, Xie MK, Wu J. Association between Ocular axial length-related genes and high myopia in a Han Chinese population. Ophthalmologica. 2016;235(1):57-60.

31. Wei WB, et al. Subfoveal choroidal thickness: the Beijing Eye Study. Ophthalmology. 2013;120(1):175-80.

32. Wang NK, et al. Choroidal thickness and biometric markers for the screening of lacquer cracks in patients with high myopia. PLoS One. 2013;8(1):e53660.

33. Wang S, et al. Choroidal thickness and high myopia: a cross-sectional study and meta-analysis. BMC Ophthalmol. 2015;15:70.

34. Tokoro T. Types of fundus changes in the posterior pole. In: Atlas of posterior fundus changes in pathologic myopia. Tokyo: Springer; 1998. p. 5-22.

35. Ohno-Matsui K. Myopic chorioretinal atrophy. In: Spaide RF, Ohno-Matsui K, Yannuzzi LA, editors. Pathologic myopia. New York: Springer; 2014. p. 187-209.

36. Ohno-Matsui K, et al. International photographic classification and grading system for myopic maculopathy. Am J Ophthalmol. 2015;159(5):877-83.e7.

37. Jonas JB, et al. Bruch membrane and the mechanism of myopization: a new theory. Retina. 2017;37(8):1428-40.

38. Ohno-Matsui K, Jonas JB, Spaide RF. Macular bruch membrane holes in highly myopic patchy chorioretinal atrophy. Am J Ophthalmol. 2016;166:22-8.

39. Ohno-Matsui K, Tokoro T. The progression of lacquer cracks in pathologic myopia. Retina. 1996;16(1):29-37.

40. Pruett RC, Weiter JJ, Goldstein RB. Myopic cracks, angioid streaks, and traumatic tears in Bruch's membrane. Am J Ophthalmol. 1987;103(4):537-43.

41. Grossniklaus HE, Green WR. Pathologic findings in pathologic myopia. Retina. 1992;12(2):127-33.

42. Bai HX, et al. Bruch's membrane thickness in relationship to axial length. PLoS One. 2017;12(8):e0182080.

43. Puliafito CA, et al. Imaging of macular diseases with optical coherence tomography. Ophthalmology. 1995;102(2):217-29.

44. Tan CS, Ngo WK, Cheong KX. Comparison of choroidal thicknesses using swept source and spectral domain optical coherence tomography in diseased and normal eyes. Br J Ophthalmol. 2015;99(3):354-8.

45. Tan CS, et al. Comparison of retinal thicknesses measured using swept-source and spectraldomain optical coherence tomography devices. Ophthalmic Surg Lasers Imaging Retina. 2015;46(2):172-9.

46. Alshareef RA, et al. Subfoveal choroidal vascularity in myopia: evidence from spectral-domain optical coherence tomography. Ophthalmic Surg Lasers Imaging Retina. 2017;48(3):202-7.

47. Takase N, et al. Enlargement of foveal avascular zone in diabetic eyes evaluated by en face optical coherence tomography angiography. Retina. 2015;35(11):2377-83.

48. Tan CS, et al. Optical coherence tomography angiography evaluation of the parafoveal vasculature and its relationship with ocular factors. Invest Ophthalmol Vis Sci. 2016;57(9):224-34.

49. Shinohara K, et al. Ultrawide-field OCT to investigate relationships between myopic macular retinoschisis and posterior staphyloma. Ophthalmology. 2018;125:1575-86.

50. Baum G, Greenwood I. The application of ultrasonic locating techniques to ophthalmology. II. Ultrasonic slit lamp in the ultrasonic visualization of soft tissues. AMA Arch Ophthalmol. 1958;60(2):263-79. 
51. Coleman DJ, et al. High-resolution ultrasonic imaging of the posterior segment. Ophthalmology. 2004;111(7):1344-51.

52. Reinstein DZ, Silverman RH, Coleman DJ. High-frequency ultrasound measurement of the thickness of the corneal epithelium. Refract Corneal Surg. 1993;9(5):385-7.

53. Hewick SA, et al. A comparison of $10 \mathrm{MHz}$ and $20 \mathrm{MHz}$ ultrasound probes in imaging the eye and orbit. Br J Ophthalmol. 2004;88(4):551-5.

54. Silverman RH, et al. Pulse-encoded ultrasound imaging of the vitreous with an annular array. Ophthalmic Surg Lasers Imaging. 2012;43(1):82-6.

55. Van Reeth E, et al. Isotropic reconstruction of a 4-D MRI thoracic sequence using superresolution. Magn Reson Med. 2015;73(2):784-93.

56. Milanfar P. Super-resolution imaging. Boca Raton: CRC Press; 2010.

57. Collins MJ, Buehren T, Iskander DR. Retinal image quality, reading and myopia. Vis Res. 2006;46(1-2):196-215.

58. Pope JM, et al. Three-dimensional MRI study of the relationship between eye dimensions, retinal shape and myopia. Biomed Opt Express. 2017;8(5):2386-95.

59. Kuo AN, et al. Posterior eye shape measurement with retinal OCT compared to MRI. Invest Ophthalmol Vis Sci. 2016;57(9):196-203.

60. Ng DS, et al. Advances of optical coherence tomography in myopia and pathologic myopia. Eye. 2016;30(7):901-16.

61. Ang M, et al. Optical coherence tomography angiography: a review of current and future clinical applications. Graefes Arch Clin Exp Ophthalmol. 2018;256(2):237-45.

62. Tan ACS, et al. An overview of the clinical applications of optical coherence tomography angiography. Eye. 2018;32(2):262-86.

63. Ang M, et al. Optical coherence tomography angiography and indocyanine green angiography for corneal vascularisation. Br J Ophthalmol. 2016;100(11):1557-63.

64. Realini $\mathrm{T}$, et al. Normative databases for imaging instrumentation. J Glaucoma. 2015;24(6):480-3.

65. Hashemi $\mathrm{H}$, et al. Global and regional estimates of prevalence of refractive errors: systematic review and meta-analysis. J Curr Ophthalmol. 2018;30(1):3-22.

66. Kasahara K, et al. Characteristics of higher-order aberrations and anterior segment tomography in patients with pathologic myopia. Int Ophthalmol. 2017;37(6):1279-88.

67. Chansangpetch S, et al. Impact of myopia on corneal biomechanics in glaucoma and nonglaucoma patients. Invest Ophthalmol Vis Sci. 2017;58(12):4990-6.

68. Schehlein EM, Novack G, Robin AL. New pharmacotherapy for the treatment of glaucoma. Expert Opin Pharmacother. 2017;18(18):1939-46.

69. Chan C, et al. Validation of an objective scoring system for forme fruste keratoconus detection and post-LASIK Ectasia Risk Assessment in Asian eyes. Cornea. 2015;34(9):996-1004.

70. Lu Y, et al. Four-year follow-up of the changes in anterior segment after phakic collamer lens implantation. Am J Ophthalmol. 2017;178:140-9.

71. Chong EW, Mehta JS. High myopia and cataract surgery. Curr Opin Ophthalmol. 2016;27(1):45-50.

72. Ang M, et al. Anterior segment optical coherence tomography. Prog Retin Eye Res. 2018;66(9):132-56.

73. Ohno-Matsui K. What is the fundamental nature of pathologic myopia? Retina. 2017;37(6):1043-8.

74. Tanaka Y, et al. Retromode retinal imaging of macular retinoschisis in highly myopic eyes. Am J Ophthalmol. 2010;149(4):635-640.e1.

75. Johnson MW. Myopic traction maculopathy: pathogenic mechanisms and surgical treatment. Retina. 2012;32(Suppl 2):S205-10.

76. Wong $\mathrm{CW}$, et al. Is choroidal or scleral thickness related to myopic macular degeneration? Invest Ophthalmol Vis Sci. 2017;58(2):907-13.

77. Shinohara K, et al. Characteristics of peripapillary staphylomas associated with high myopia determined by swept-source optical coherence tomography. Am J Ophthalmol. 2016;169:138-44. 
78. Shinohara K, et al. Posterior staphylomas in pathologic myopia imaged by widefield optical coherence tomography. Invest Ophthalmol Vis Sci. 2017;58(9):3750-8.

79. Ohno-Matsui K, et al. Features of posterior staphylomas analyzed in wide-field fundus images in patients with unilateral and bilateral pathologic myopia. Retina. 2017;37(3):477-86.

80. Iacono $\mathrm{P}$, et al. Fluorescein angiography and spectral-domain optical coherence tomography for monitoring anti-VEGF therapy in myopic choroidal neovascularization. Ophthalmic Res. 2014;52(1):25-31.

81. Kaneko Y, et al. Areas of nonperfusion in peripheral retina of eyes with pathologic myopia detected by ultra-widefield fluorescein angiography. Invest Ophthalmol Vis Sci. 2014;55(3):1432-9.

82. Miyata M, et al. Detection of myopic choroidal neovascularization using optical coherence tomography angiography. Am J Ophthalmol. 2016;165:108-14.

83. Bruyere E, et al. Neovascularization secondary to high myopia imaged by optical coherence tomography angiography. Retina. 2017;37(11):2095-101.

84. Querques G, et al. Optical coherence tomography angiography of choroidal neovascularization secondary to pathologic myopia. Dev Ophthalmol. 2016;56:101-6.

85. Querques L, et al. Optical coherence tomography angiography of myopic choroidal neovascularisation. Br J Ophthalmol. 2017;101(5):609-15.

86. Ohno-Matsui K, et al. Updates of pathologic myopia. Prog Retin Eye Res. 2016;52:156-87.

87. Battaglia Parodi M, Iacono P, Bandello F. Correspondence of leakage on fluorescein angiography and optical coherence tomography parameters in diagnosis and monitoring of myopic choroidal neovascularization treated with bevacizumab. Retina. 2016;36(1):104-9.

88. Ohno-Matsui K, Jonas JB, Spaide RF. Macular bruch membrane holes in choroidal neovascularization-related myopic macular atrophy by swept-source optical coherence tomography. Am J Ophthalmol. 2016;162:133-139.e1.

89. Ishida $\mathrm{T}$, et al. Possible connection of short posterior ciliary arteries to choroidal neovascularisations in eyes with pathologic myopia. Br J Ophthalmol. 2018;103(4):457-62.

90. Hayashi $\mathrm{K}$, et al. Value of indocyanine green angiography in the diagnosis of occult choroidal neovascular membrane. Jpn J Ophthalmol. 1988;42:827-9.

91. Geeraets WJ, Berry ER. Ocular spectral characteristics as related to hazards from lasers and other light sources. Am J Ophthalmol. 1968;66(1):15-20.

92. Spaide RF, Koizumi H, Pozonni MC. Enhanced depth imaging spectral-domain optical coherence tomography. Am J Ophthalmol. 2008;146(4):496-500.

93. Choma MA, et al. Sensitivity advantage of swept source and Fourier domain optical coherence tomography. Opt Express. 2003;11(18):2183-9.

94. Chung CW, et al. Enhancement of corneal visibility in optical coherence tomography images with corneal opacification. Transl Vis Sci Technol. 2016;5(5):3.

95. Girard MJ, et al. Enhancement of corneal visibility in optical coherence tomography images using corneal adaptive compensation. Transl Vis Sci Technol. 2015;4(3):3.

96. Alkabes M, Mateo C. Macular buckle technique in myopic traction maculopathy: a 16-year review of the literature and a comparison with vitreous surgery. Graefes Arch Clin Exp Ophthalmol. 2018;256(5):863-77.

97. Wollensak G, Iomdina E. Long-term biomechanical properties of rabbit sclera after collagen crosslinking using riboflavin and ultraviolet A (UVA). Acta Ophthalmol. 2009;87(2):193-8.

98. Kim M, et al. Pharmacologic alternatives to riboflavin photochemical corneal cross-linking: a comparison study of cell toxicity thresholds. Invest Ophthalmol Vis Sci. 2014;55(5):3247-57.

99. Chang RT, Singh K. Myopia and glaucoma: diagnostic and therapeutic challenges. Curr Opin Ophthalmol. 2013;24(2):96-101.

100. Jonas JB, Gusek GC, Naumann GO. Optic disk morphometry in high myopia. Graefes Arch Clin Exp Ophthalmol. 1988;226(6):587-90.

101. Jonas JB, et al. Intraocular pressure and glaucomatous optic neuropathy in high myopia. Invest Ophthalmol Vis Sci. 2017;58(13):5897-906.

102. Ohno-Matsui $\mathrm{K}$, et al. Long-term development of significant visual field defects in highly myopic eyes. Am J Ophthalmol. 2011;152(2):256-265.e1. 
103. Han JC, et al. The characteristics of deep optic nerve head morphology in myopic normal tension glaucoma. Invest Ophthalmol Vis Sci. 2017;58(5):2695-704.

104. Sawada Y, et al. Multiple temporal lamina cribrosa defects in myopic eyes with glaucoma and their association with visual field defects. Ophthalmology. 2017;124(11):1600-11.

105. Malik R, et al. Diagnostic accuracy of optical coherence tomography and scanning laser tomography for identifying glaucoma in myopic eyes. Ophthalmology. 2016;123(6):1181-9.

106. Enders P, et al. Novel Bruch's membrane opening minimum rim area equalizes disc size dependency and offers high diagnostic power for glaucoma. Invest Ophthalmol Vis Sci. 2016;57(15):6596-603.

107. Nakanishi H, et al. Sensitivity and specificity for detecting early glaucoma in eyes with high myopia from normative database of macular ganglion cell complex thickness obtained from normal non-myopic or highly myopic Asian eyes. Graefes Arch Clin Exp Ophthalmol. 2015;253(7):1143-52.

108. Hung KC, et al. Macular diagnostic ability in OCT for assessing glaucoma in high myopia. Optom Vis Sci. 2016;93(2):126-35.

109. Akashi A, et al. The ability of macular parameters and circumpapillary retinal nerve fiber layer by three SD-OCT instruments to diagnose highly myopic glaucoma. Invest Ophthalmol Vis Sci. 2013;54(9):6025-32.

110. Mwanza JC, et al. Diagnostic performance of optical coherence tomography ganglion cell--inner plexiform layer thickness measurements in early glaucoma. Ophthalmology. 2014;121(4):849-54.

111. Zhang Y, Wen W, Sun X. Comparison of several parameters in two optical coherence tomography systems for detecting glaucomatous defects in high myopia. Invest Ophthalmol Vis Sci. 2016;57(11):4910-5.

112. Suwan Y, et al. Association of myopia with peripapillary perfused capillary density in patients with glaucoma: an optical coherence tomography angiography study. JAMA Ophthalmol. 2018;136(5):507-13.

113. Summers JA. The choroid as a sclera growth regulator. Exp Eye Res. 2013;114:120-7.

114. Campbell IC, Coudrillier B, Ross Ethier C. Biomechanics of the posterior eye: a critical role in health and disease. J Biomech Eng. 2014;136(2):021005.

115. Kiss B, et al. Ocular hemodynamics during isometric exercise. Microvasc Res. 2001;61(1):1-13.

116. Ferrara D, Waheed NK, Duker JS. Investigating the choriocapillaris and choroidal vasculature with new optical coherence tomography technologies. Prog Retin Eye Res. 2016;52:130-55.

117. Spaide RF. Choriocapillaris flow features follow a power law distribution: implications for characterization and mechanisms of disease progression. Am J Ophthalmol. 2016;170:58-67.

118. Zhang Q, et al. A novel strategy for quantifying choriocapillaris flow voids using sweptsource OCT angiography. Invest Ophthalmol Vis Sci. 2018;59(1):203-11.

119. Al-Sheikh M, et al. Quantitative OCT angiography of the retinal microvasculature and the choriocapillaris in myopic eyes. Invest Ophthalmol Vis Sci. 2017;58(4):2063-9.

120. Ang M, et al. Comparison of anterior segment optical coherence tomography angiography systems for corneal vascularisation. Br J Ophthalmol. 2018;102(7):873-7.

121. Cai Y, et al. Serial optical coherence tomography angiography for corneal vascularization. Graefes Arch Clin Exp Ophthalmol. 2017;255(1):135-9.

122. Ang M, et al. En face optical coherence tomography angiography for corneal neovascularisation. Br J Ophthalmol. 2016;100(5):616-21.

123. Grudzi E, et al. Modern diagnostic techniques for the assessment of ocular blood flow in myopia: current state of knowledge. J Ophthalmol. 2018;2018:8.

124. Silverman RH, et al. $75 \mathrm{MHz}$ ultrasound biomicroscopy of anterior segment of eye. Ultrason Imaging. 2006;28(3):179-88.

125. Liu W, Zhang HF. Photoacoustic imaging of the eye: a mini review. Photoacoustics. 2016;4(3):112-23.

126. Xie D, et al. In vivo blind-deconvolution photoacoustic ophthalmoscopy with total variation regularization. J Biophotonics. 2018;11(9):e201700360. 
127. Larin KV, Sampson DD. Optical coherence elastography - OCT at work in tissue biomechanics. Biomed Opt Express. 2017;8(2):1172-202.

128. Li G-Y, Cao Y. Mechanics of ultrasound elastography. Proc Math Phys Eng Sci. 2017;473(2199):20160841.

129. Karyotakis NG, et al. Manometric measurement of the outflow facility in the living human eye and its dependence on intraocular pressure. Acta Ophthalmol. 2015;93(5):e343-8.

130. Beaton L, et al. Non-invasive measurement of choroidal volume change and ocular rigidity through automated segmentation of high-speed OCT imaging. Biomed Opt Express. 2015;6(5):1694-706.

131. Sit AJ, et al. In vivo noninvasive measurement of young's modulus of elasticity in human eyes: a feasibility study. J Glaucoma. 2017;26(11):967-73.

132. Zaitsev VY, et al. Optical coherence elastography for strain dynamics measurements in laser correction of cornea shape. J Biophotonics. 2017;10(11):1450-63.

133. O'Hara KE, et al. Measuring pulse-induced natural relative motions within human ocular tissue in vivo using phase-sensitive optical coherence tomography. J Biomed Opt. 2013;18(12):121506.

134. Dragostinoff N, et al. Time course and topographic distribution of ocular fundus pulsation measured by low-coherence tissue interferometry. J Biomed Opt. 2013;18(12):121502.

135. Qu Y, et al. In vivo elasticity mapping of posterior ocular layers using acoustic radiation force optical coherence elastography. Invest Ophthalmol Vis Sci. 2018;59(1):455-61.

Open Access This chapter is licensed under the terms of the Creative Commons Attribution 4.0 International License (http://creativecommons.org/licenses/by/4.0/), which permits use, sharing, adaptation, distribution and reproduction in any medium or format, as long as you give appropriate credit to the original author(s) and the source, provide a link to the Creative Commons license and indicate if changes were made.

The images or other third party material in this chapter are included in the chapter's Creative Commons license, unless indicated otherwise in a credit line to the material. If material is not included in the chapter's Creative Commons license and your intended use is not permitted by statutory regulation or exceeds the permitted use, you will need to obtain permission directly from the copyright holder.

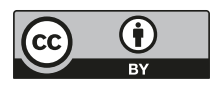

